\title{
Effect of ovarian types and collection techniques on the number of follicles and the quality of cumulus-oocyte-complexes in cow
}

\author{
Khandoker MAMY*, NF Atiqah and N Ariani
}

Faculty of Sustainable Agriculture, Universiti Malaysia Sabah, Beg Berkunci No. 3, 90509 Sandakan, Sabah, Malaysia

\begin{abstract}
This experiment was conducted for evaluation of bovine slaughterhouse ovary, follicles, and cumulusoocyte-complexes (COCs) and to compare the effect of collection techniques on the recovery rate of COCs. The collected slaughterhouse ovaries was classified as corpus luteum present $(\mathrm{CL}+)$ and corpus luteum absent (CL-) groups. It was found that $62.5 \%$ of the ovaries collected were CL- type and $37.5 \%$ were $\mathrm{CL}+$ type. For collection of COCs, blunt dissection and aspiration techniques were performed and number of follicles collected was recorded. The higher number of follicles were dissected and aspirated from $C L$ - ovaries (11.2 \pm 1.8 and $37.8 \pm 14.9$, respectively) compared to $C L+$ ovaries $10.5 \pm 1.5$ and $28.3 \pm 15.6$, respectively). The follicular materials collected from both techniques were observed under microscope to categorize the COCs as A (oocyte surrounded with cumulous cells homogenously), B (oocyte surrounded with cumulous cells partially), C (oocyte not surrounded at all by cumulous cells) and $D$ (degeneration observed both in oocyte and cumulous cells). Grade A and grade B were classified as normal and grade $C$ and grade $D$ were considered as abnormal COCs. The result indicated that ovaries having no $\mathrm{CL}$ contributing more total number of COCs per ovary (6.8 \pm 1.0$)$ and also contributing higher normal COCs $(5.7 \pm 0.9)$ than that of ovaries with $C L(6.0 \pm 2.0$ and $4.5 \pm 1.5$, respectively) in blunt dissection technique. But same trend of result was not found in aspiration technique. Similarly, higher percentage of COCs recovery rate was also recorded in blunt dissection $(61.6 \pm 4.6 \%$ vs $16.5 \pm 4.9 \%$, on total basis) than aspiration ( $48.6 \pm 2.9 \%$ vs $11.7 \pm 4.1 \%$, on normal basis) technique. So, ovaries without $\mathrm{CL}$ and blunt dissection technique found more suitable for harvesting the higher number and superior quality of COCs for extending the in vitro embryo production experiment.
\end{abstract}

Key words: slaughterhouse ovary, corpus luteum, blunt dissection, aspiration

Bangladesh Animal Husbandry Association. All rights reserved.

Bang. J. Anim. Sci. 2016. 45 (3):10-16

\section{Introduction}

The cow is usually produces a single ovum during the period of ovulation. If the ovum is fertilized in vivo, a cow only delivers a calf after an average of nine months of gestation (Hafez, 1993) and this situation causes slow genetic improvement. Hence, over a decade, there were a lot of researches done towards the implementation of embryo technologies to fasten the genetic manipulation of livestock which involves multiple ovulation and embryo transfer (MOET), in vitro embryo production (IVEP), cloning, and transgenesis (Asad, 2015; Sahaet al., 2014; Sreenivaset al., 2014; Freitas and Melo, 2010; Moadalet al., 2008; Hyttelet al., 1997; Wang et al., 2007; Sianturi, 2001; Sirad and Blondin, 1996).
From those mentioned, IVEP has becoming more popular method of producing embryos from slaughter house derived ovaries (Hoqueet al., 2011). The IVEP system involves at least four steps, namely (i) evaluation of ovaries, efficient collection and grading of oocytes; (ii) in vitro maturation (IVM) of these oocytes; (iii) in vitro fertilization (IVF) of the matured oocytes; and (iv) in vitro culture (IVC) of the resulting embryos (Freitas and Melo, 2010). Nowadays, IVEP is becoming a useful tool for maximizing the number of offspring from valuable cows, producing calves from infertile and slaughtered cows, and producing commercial beef cattle production program without brood cows (Sianturi, 2001; Hyttel et al., 1997; Sirad and Blondin, 1996). In Malaysia, very limited research so far has been done on in vitro production of embryos

*Corresponding author: mamyahiak@ums.edu.my 
from slaughterhouse derived cattle oocytes (Sianturi, 2001).

To produce embryos by in vitro techniques, it is necessary to recover the oocytes and undergo maturation of oocytes, fertilize, and develop those developing zygotes to blastocyst stage so that they can be transferred to the recipient (Hoque et al., 2012).COCs contains the oocytes and in recent years, the percentage of oocytes reaching the blastocyst stage by in vitro techniques still varies. In some cases, the low development of IVM oocytes is related to their quality at the beginning of maturation. Mondal (2008) has reported that higher average number of good quality oocytes was recovered from ovaries without corpus luteum compared to the ovaries with corpus luteum, which thus, can be effectively used for IVM and IVF. In addition, it has been shown that oocytes with at least four layers of cumulus cells have good result for IVM and IVF (Yang et al., 1993).

There is a huge gap between the demand and supply of milk and meat in Malaysia (Jamaludinet al., 2014) due to small cattle population and low productivity. In Malaysia, there is still no information so far has been found on evaluation of bovine slaughterhouse ovaries, follicles, and cumulus-oocyte-complexes (COCs) for IVEP. Moreover, in Sabah, it is really difficult to get remarkable number of slaughterhouse ovaries to collect COCs for initiating the embryo production experiment as rarely slaughtered cow in this area. So, for successful IVEP experiment in cowfor the evaluation of ovaries it is important to find out the efficient collection techniques of COCs. Therefore, present study was performed as an initial work for planning and execution of future pragmatic research on in vitro production of bovine embryos to increase cattle productivity in Sabah as well as in Malaysia. The main purpose of this research is to determine the optimum type of ovary (corpus luteum present or absent) and COCs recovery technique that is most suitable to produce higher number of good quality COCs for IVEP experiment.

\section{Materials and Methods}

The experiment was conducted at the Anatomy and Physiology Laboratory, Faculty of Sustainable Agriculture, Universiti Malaysia Sabah (UMS)
Sandakan Campus from July 2015 to September 2015.

\section{Collection, processing and evaluation of ovaries}

Cow's ovaries with unknown reproductive history were collected from Hafiz Farm Slaughterhouse at LahadDatu, Sabah and put in falcon tube containing $0.9 \%$ physiological saline that was warmed at $25-30^{\circ} \mathrm{C}$ and kept in a polystyrene box to maintain this temperature during transporting the ovaries (Mondalet al., 2008). The ovaries were transported to the laboratory within 4-5 hours of slaughter. The ovaries were then transferred to sterilized Petri dish and rinsed thoroughly by physiological saline at $25^{\circ} \mathrm{C}$. Each ovary was trimmed to remove the surrounding tissues and overlying bursa.The ovaries were then observed and categorized as corpus luteum absent ( $\mathrm{CL}-)$ and corpus luteum present $(\mathrm{CL}+$ ) groups (Figure 1 ) and the number of both types of ovaries were recorded (Mondalet al., 2008). The follicles on the surface of the ovary for dissection or aspiration were counted and data was recorded.

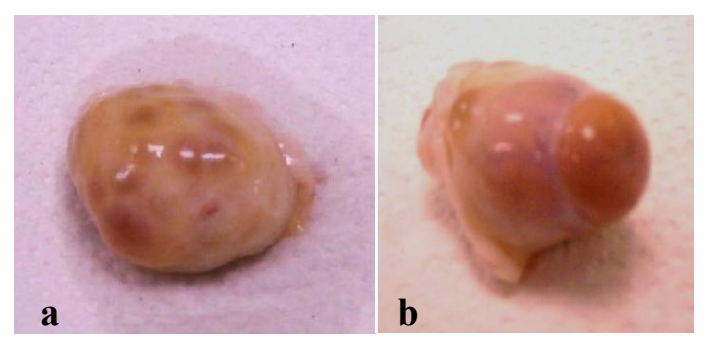

Figure1. Ovaries without corpus luteum (a) and ovaries with corpus luteum (b)

\section{Collection and grading of cumulus-oocyte- complexes (COCs)}

Two collection techniques were applied in thisstudy which was blunt dissection and aspiration techniques. For blunt dissection technique, individual follicles were dissected from the ovaries by using scissors and forceps \{Figure 2 (a) \}and the number was recorded. Then, the follicular material was harvested individually in the Petri dish. A small amount of physiological saline solution $(0.9 \% \mathrm{NaCl})$ with $5 \%$ bovine serum albumin (BSA) was added on the slides with follicular materials collected, followed by observing under microscope to grade the COCs. 

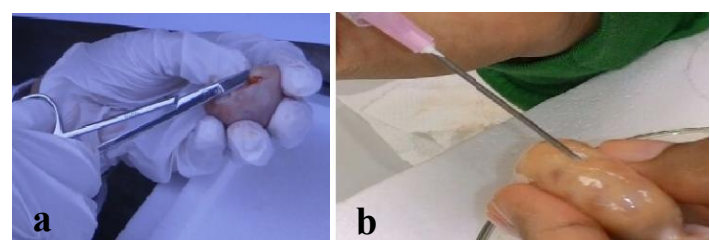

Figure 2. Representative photograph showing blunt dissection (a) and aspiration (b) techniques

For aspiration technique, $10-\mathrm{ml}$ syringe was loaded with $0.9 \%$ physiological solution (1.0 to $1.5 \mathrm{ml}$ ), and $18 \mathrm{G}$ needle was put in the ovarian parenchyma near the vesicular follicles and all visible follicles in the ovarian surface were aspirated fFigure 2 (b)\}. After that, the aspirated follicular materials were transferred slowly into a falcon tube to avoid damage of the cumulus cells and the numbers of follicles aspirated were also recorded.
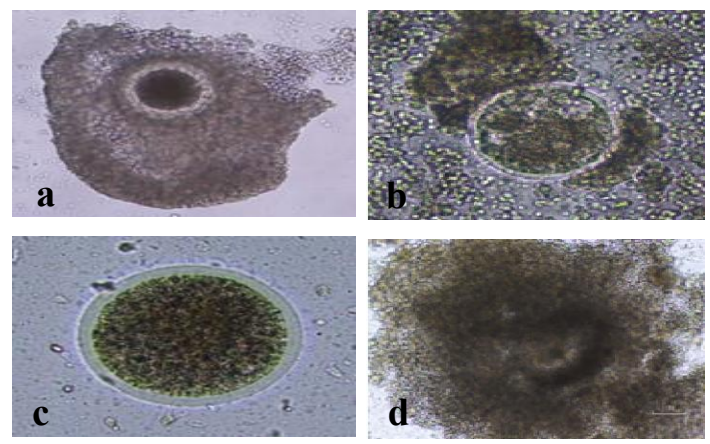

Figure 3. Grading of collected COCs; (a) Grade-A; (b) Grade-B; (c) Grade-C and (d) Grade-D

\section{Statistical analysis}

The data collected was compiled, tabulated and analysed in accordance with the objectives and descriptive statistics was performed with the help of Microsoft Office Excel 2007.

\section{Results}

Quantitative evaluation of bovine
slaughterhouse ovaries

Bovine ovaries were collected from local slaughterhouse and were classified into two groups which were with corpus luteum $(\mathrm{CL}+)$ and without corpus luteum (CL-). Among 16 ovaries collected, 10 were found as CL- ovaries and 6 as $\mathrm{CL}+$. It was found that $62.5 \%$ of the ovaries collected were CL- type and $37.5 \%$ were $\mathrm{CL}+$.

\section{Quantitative and qualitative evaluation of follicles and COCs using blunt dissection and aspiration techniques}

\section{Blunt dissection technique}

The results for number of dissectible follicles on the surface of the ovary, the number of follicles dissected and the number of COCs collected is summarized in Table 1 . Total visible dissectible follicles recorded on the surface of both types of ovaries were 115. Among them 93 were recorded in 6 without corpus luteum ovaries and 22 in 2 with corpus luteum ovaries. On an average, $15.5 \pm 2.7$ dissectible follicles per ovary were found in CL- group and $11.0 \pm 2.0$ dissectible follicles per ovary were found in $\mathrm{CL}+$ group. From Table 1, it shows that a total of 88 follicles were dissected from both types of ovaries and among them 67 obtained from $6 \mathrm{CL}$ - ovaries and 21 from $2 \mathrm{CL}+$ ovaries. Based on the mean value, it is shown that number of follicles dissected from CLwas higher $(11.2 \pm 1.8)$ than that of $\mathrm{CL}+$ $(10.5 \pm 1.5)$ ovaries. Similarly, the higher number of total COCs per ovary was collected from CLgroup (6.8 \pm 1.0$)$ than in $C L+$ group (6.0 \pm 2.0$)$.

Table 1. Types of ovary and number of follicles and cumulus-oocyte-complexes per ovary using blunt dissection technique*

\begin{tabular}{lcccccc}
\hline Ovarian type & $\begin{array}{c}\text { Total number } \\
\text { of dissectible } \\
\text { follicle/ ovary } \\
\text { (mean } \pm \text { SE) }\end{array}$ & $\begin{array}{c}\text { Follicle } \\
\text { dissected/ } \\
\text { ovary } \\
\text { (mean } \pm \text { SE) }\end{array}$ & Normal & Abnormal & Total \\
\hline Ovary without CL (6) & $15.5 \pm 2.7(93)$ & $11.2 \pm 1.8(67)$ & $5.7 \pm 0.9(34)$ & $1.2 \pm 0.5(7)$ & $6.8 \pm 1.0(41)$ \\
Ovary with CL (2) & $11.0 \pm 2.0(22)$ & $10.5 \pm 1.5(21)$ & $4.5 \pm 1.5(9)$ & $1.5 \pm 0.5(3)$ & $6.0 \pm 2.0(12)$ \\
\hline
\end{tabular}

*Parentheses indicates total numbers 
Then, when the COCs were classified into normal and abnormal, higher number of normal (grade $A$ and $B$ ) COCs were found in CL- group ovaries (5.7 \pm 0.9$)$ than that of $\mathrm{CL}+$ group ovaries $(4.5 \pm 1.5)$ while reverse trend of result was found in abnormal with respective mean of 1.2 and 1.5 cocs per ovary (Table 1 ).

\section{Aspiration technique}

An alternative aspiration technique was also adopted in this experiment for collecting of COCs and the results are summarized in Table 2 . In this technique, total aspirable follicles observed in both types of ovaries were 323 from 8 ovaries and among them 180 was found in $4 \mathrm{CL}$ - group ovaries and 143 in $4 \mathrm{CL}+$ group ovaries. Again average of $45.0 \pm 14.3$ follicles per ovary was recorded in CL- group and $35.8 \pm 14.4$ follicles per ovary were recorded in $\mathrm{CL}+$ group. Table 2 further shows that higher number of follicles aspirated per ovary in CL- group (37.8 \pm 14.9$)$ than that of $\mathrm{CL}+$ group $(28.3 \pm 15.6)$. On the other hand, a little higher number of total COCs was collected from $\mathrm{CL}+$ group $(5.3 \pm 2.3)$ than that of CL- group (4.5 \pm 1.4$)$. Similarly, higher number of normal COCs $(4.8 \pm 2.0)$ per ovary was obtained from $\mathrm{CL}+$ ovaries than that of $\mathrm{CL}$ ovaries (2.8 \pm 1.1$)$. Both findings are unusual and similar unusual result also found in abnormal COCs, with the mean of COCs per ovary is 0.5 in $\mathrm{CL}+$ ovary and 1.8 in CL- ovary (Table 3 ).

\section{Comparison of blunt dissection and aspiration techniques}

To compare the blunt dissection and aspiration techniques, the results of COCs recovery rate was calculated according the formula of AbdelKhalehet al. (2010) and the result is summarized in Table 3. From Table 3, it is clear that among the total COCs recovery $(61.6 \pm 4.6 \%)$, $48.6 \pm 2.9 \%$ normalCOCs recovery rate were found higher in blunt dissection technique compared to aspiration technique (16.5 $\pm 4.9 \%$ and $11.7 \pm 4.1 \%$ total and normal, respectively). When comparing these two methods on the basis of abnormal COCs recovery rate, again higher recovery rate of COCs was recorded in blunt dissection technique $(13.0 \pm 3.4)$ than that of aspiration technique $(4.8 \pm 2.6 \%)$.

\section{Discussion}

\section{Evaluation of bovine slaughterhouse ovaries}

From the observation of this study, it was shown that among the 16 ovaries collected from slaughterhouse, 10 ovaries were obtained as without corpus luteum (62.5\%) and 6 ovaries were obtained as with corpus luteum (37.5\%). The numbers of ovaries having no corpus luteum usually obtaining from non-cyclic cows were slaughtered for economic reason. Commonly, less reproductive performing cows were slaughtered and cause high possibility to get more CL- ovaries from the slaughterhouse during sample collection.

The same trend of result was also reported in buffaloes by Khandoker et al. (2011) where among 136 ovaries, 93 were found without CL and the remaining 43 ovaries with CL. Similar results also reported in goat (Asad, 2015; Saha et al., 2014; Mondalet al.,2008 and Islam et al. (2007). Those findings further supported the statement that for economic reason, typically less reproductive performing cows are slaughtered in slaughterhouse and most of them might be non-

Table 2. Types of ovary and number of follicles and cumulus-oocyte-complexes per ovary using aspiration technique

\begin{tabular}{l|c|l|c|c|c}
\hline Ovarian type & $\begin{array}{l}\text { Total number of } \\
\text { aspirable follicle } \\
\text { (mean } \pm \text { SE) }\end{array}$ & $\begin{array}{l}\text { Number } \\
\text { follicle aspirated } \\
\text { (mean } \pm \text { SE) }\end{array}$ & \multicolumn{3}{|c}{$\begin{array}{c}\mid c \\
\text { (mean } \pm \text { SE) }\end{array}$} \\
\cline { 4 - 6 } & & Normal & Abnormal & Total \\
\hline Ovary without CL (4) & $45.0 \pm 14.3(180)$ & $37.8 \pm 14.9(151)$ & $2.8 \pm 1.1(11)$ & $1.8 \pm 1.4(7)$ & $4.5 \pm 1.4(18)$ \\
Ovary with CL (4) & $35.8 \pm 14.5(143)$ & $28.3 \pm 15.6(113)$ & $4.8 \pm 2.0(19)$ & $0.5 \pm 0.5(2)$ & $5.3 \pm 2.3(21)$ \\
\hline
\end{tabular}


cyclic. Commonly, the older or finisher non-cyclic animals were brought for slaughtering in the slaughterhouse.

\section{Quantitative evaluation of bovine ovarian follicles}

The results obtained from this study shows that ovaries having no corpus luteum contributing larger number of follicles for dissection $(15.5 \pm 2.7)$ and aspiration $(45.0 \pm 14.3)$ than that of the ovaries with corpus luteum for dissection $(11.0 \pm 2.0)$ and aspiration (35.8 14.5$)$. The results further indicated that higher number of follicles were able to dissected and aspirated from ovaries without $\mathrm{CL}(11.2 \pm 1.8$ and $37.8 \pm 14.9$, respectively) than that of ovaries with $\mathrm{CL}$ (10.5 \pm 1.5 and $28.3 \pm 15.6$, respectively) (Table 2 and 3).The higher number of follicles found in without $\mathrm{CL}$ ovaries than those of with $\mathrm{CL}$ ovaries fits with the endocrinological explanation. The presence of corpus luteum in cyclic ovary cause a higher level of progesterone hormone production in which giving a negative response to anterior pituitary gland for the restriction of gonadotrophin secretion and leads to follicular degeneration and inhibition of the development of large follicles (Webb et al. 1999). In noncyclic female, the absence of corpus luteum cause no negative effect of progesterone on anterior pituitary and thus estrogen-progesterone levels remains balanced which allows the growth of follicles. Ginther et al., (1996) stated that ovaries without $\mathrm{CL}$, the decrease in progesterone leads to increase in $\mathrm{GnRH}$ which stimulates the release of follicle stimulating hormone (FSH) and this hormone causes the rapid growth of ovarian follicles.

The results strongly supported by the previous finding of Asad (2015), who reported that higher number of follicles aspirated per ovary in without $\mathrm{CL}$ group (2.92 \pm 0.08$)$ than those of the with $\mathrm{CL}$ group $(2.52 \pm 0.11)$ in goat. Similar findings also found in buffalo ovaries by Khandokeret al. (2011) where significantly higher number of follicles were collected in ovaries without $\mathrm{CL}$ $(6.78 \pm 0.18)$ than in $\mathrm{CL}$ containing ovaries $(4.09 \pm 0.26)$.

\section{Quantitative and qualitative evaluation of cocs}

Both the number and quality of the COCs are important to initiate the embryo culture experiment and the results of the present study clearly indicated that ovaries without corpus luteum contributing higher number of total COCs per ovary(6.8 1.0$)$ than that of with corpus luteum group ovaries $(6.0 \pm 2.0)$ at least in blunt dissection technique (Table 2 ).

From this study, it was also found that more normal ( $A$ and $B$ grade) COCs was dissected from ovaries without corpus luteum (5.7 \pm 0.9$)$ compared to ovaries with corpus luteum (4.5 \pm 1.5$)$ (Table 2). Nandi et al. (2000) stated that when ovaries had a corpus luteum, the oocyte recovery rate decreases. This is because there will be restriction of follicular development as lutein cells occupy most of the ovary (Kumar et al., 2004). Hafez (1993) mentioned that in the presence of $C L$ in ovary, the growth of follicle is inhibited while atresia is increased. These statements can be the physiological explanation for lower number of COCs in the with CL ovaries compared to without $\mathrm{CL}$ ovaries. Our finding further supported by other researchers, they have done their research in goat (Asad, 2015; Khandokeret al., 2011; Mondalet al., 2008and Islam et al., 2007).

Similarly, the higher number of normal COCs in $\mathrm{CL}$ absent group ovaries may be due to the hormonal effect of $C L$. When $C L$ is absent in the ovary, progesterone which has role in follicular degeneration could not be produced (Hafez, 1993). Thus, folliculogenesis can occur successfully and further there is more chance to produce high quality of COCs. Therefore, the types of ovary at the time of COCs collection have affected the quantity and quality of COCs recovered as well as usable oocytes in animals for use in IVEP program. A bit exception result, in respect of total COCs aspirated per ovary was found higher in $\mathrm{CL}+$ group ovaries $(5.3 \pm 2.3)$ than that of CL- group of ovaries $(4.8 \pm 2.0)$ though the total number of aspirable follicles and follicles aspirated were recorded higher in CL- group ovaries $(45.0 \pm 14.3$ vs $37.8 \pm 14.9)$ than that of $\mathrm{CL}+$ group of ovaries ( $35.8 \pm 14.5$ vs $28.3 \pm 15.6$ ). This finding further suggested that to collect COCs by aspiration technique, efficiency of the 
technician is the prerequisite factor and also reflects the superiority of blunt dissection technique once more again.

Table 3.COCs recovery rate in aspiration and blunt dissection techniques*

\begin{tabular}{llcl}
\hline $\begin{array}{l}\text { Collection } \\
\text { technique }\end{array}$ & \multicolumn{3}{c}{$\begin{array}{c}\text { Recovery rate of COCs (RR \%) } \\
\text { (mean } \pm \text { SE) }\end{array}$} \\
\cline { 2 - 4 } & Normal & Abnormal & Total \\
\hline Blunt dissection (8) & $48.6 \pm 2.9$ & $13.0 \pm 3.4$ & $61.6 \pm 4.6$ \\
Aspiration (8) & $11.7 \pm 4.1$ & $4.8 \pm 2.6$ & $16.5 \pm 4.9$ \\
\hline
\end{tabular}

*Parentheses indicates total numbers

\section{Comparison of blunt dissection and aspiration techniques}

Various techniques can be used to collect the oocytes from the ovaries and the number of oocytes recovered can be influenced by the technique used to harvest the oocytes. From the result obtained in this study, when comparing the techniques of collecting the COCs, blunt dissection technique was found more efficient than that of aspiration technique on the harvesting of higher number of COCs $(61.6 \pm 4.6 \%$ vs $16.5 \pm 4.9 \%)$. Blunt dissection technique also recovered higher rate of normal

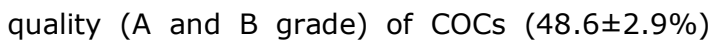
than that of aspiration technique $(11.7 \pm 4.1 \%)$. The statements are in agreement with similar study done in goat by Singh et al. (2013) where the number of oocytes retrieved was significantly higher in dissection method $(2.52 \pm 0.28)$ than aspiration $(1.35 \pm 0.18)$. Less number of COCs retrieved by aspiration technique might be because aspiration disrupts cumulus cells or damages the whole oocytes (Nowshari, 2004). Shiraziet al. (2005) also reported high number of denuded oocytes recovered through aspiration.

It is observed that aspiration technique requiring less time on harvesting the COCs compared to blunt dissection technique. However, blunt dissection technique is found more efficient for COCs retrieval with higher number and better quality of recovered COCs.Blunt dissection technique seems to be more practical in a place like Sabah where slaughterhouse ovaries getting is really difficult. This is because when comparison of follicles collected and COCs recovery from each ovary is evaluated (recovery rate of COCs), it was found that, in blunt dissection technique, the COCs recovery was averagely more than $50 \%$ than that of the aspiration technique, the COCs recovered was found not even one-third of follicles aspirated. Since more than half of the total follicles could contributesCOCs, it shows that there is high possibility to get more number of COCs by blunt dissection technique although the number of sample is less.Besides that, from the results obtained, it can be seen that blunt dissection technique does not require expertise and needful skills compared to aspiration technique. Anyone can perform blunt dissection technique to collect cOCs though the person is inexperienced in handling such process. Thus, by comparing the techniques, blunt dissection technique was found to be more efficient than that of the aspiration technique in terms of number as well as good quality of COCs collection.

\section{Conclusion}

From the results, it can be concluded that comparatively higher number of ovaries were found without corpus luteum compared to ovaries with corpus luteum showing that usually noncyclic cows are slaughtered in the slaughterhouse due to economic reason. Ovaries without CL contributing larger number of follicles dissected andaspirated per ovary compared to ovaries with CL. Furthermore, comparatively higher number of total COCs and superior quality of COCs ( $A$ and $B$ grade) were possible to obtain from without $C L$ ovaries, suggested to be suitable for collecting COCs for initiating in vitroembryo production experiment in cow. On the other hand, blunt dissection technique was found to be more efficient than aspiration technique for COCs collection and this technique seems to be more practical in the place where getting slaughterhouse ovaries is difficult.

\section{Acknowledgement}

The first author is very much thankful to Universiti Malaysia Sabah (UMS) for supporting the project and providing the fund from "SKIM PENSYARAH LANTIKAN BARU". We are also acknowledging the Faculty of Sustainable Agriculture, UMS for all sorts of logistic support, Hafiz Farm SdnBhd for providing cow ovaries. 


\section{References}

Abdel-Khalek EA, MA El-Harairy, SM Shamiah and WA Khalil (2010).Effect of ovary preservation period on recovery rate and categories of dromedary camel oocytes. Saud. J. Biol. Sci. 17:231-235

Asad LY (2015).Effect of bovine serum albumin and follicular fluid on in vitro maturation, fertilization and development of goat embryos.Ph D Thesis.Department of Animal Breeding and Genetics, Bangladesh Agricultural University.

Freitas VJF and LM Melo (2010). In vitro embryo production in small ruminants. RevistaBrasileira de Zootecnia 39: 404-413.

Ginther OJ, DR Bergfelt, LJ Kulick and K Kot (1996). Selection of the dominant follicle in cattle: role of two-way functional coupling between folliclestimulating hormone and the follicles. BiolReprod. 62: 920-927.

Hafez ESE (1993). Reproduction in Farm Animals.Fifth Edition. Lea and Febriger: Philadelphia.

Hoque SAM, SK Kabiraj, MAMY Khandoker, A Mondal and KMA Tareq (2011).Effect of collection techniques on cumulus oocyte complexes (COCs)recovery, invitro maturation and fertilization of goat oocytes.Afr. J. Biotech. 10: 9177-9181.

Hoque SAM, MAMY Khandoker, SK Kabiraj, LY Asad, M Fakruzzaman and KMA Tareq (2012). Effect of goat follicular fluid on in vitro production of embryos in Black Bengal goats.J. Appl. Anim. Sci. 2: 287-294.

Hyttel P, T Fair, H Callesen and T Greve (1997). Oocyte growth, capacitation and final maturation in cattle.Theriogenogy. 47: 23-32.

Islam MR, MAMY Khandoker, S Afroz, MGM Rahman and RI Khan, (2007). Qualitative and quantitative analysis of goat ovaries, follicles and oocytes inview of in vitro production of embryos.J. Zhejiang University. 8: 465-469.

Jamaludin $\mathrm{MH}, \mathrm{MH}$ Hassan, $\mathrm{MR}$ Amin and $\mathrm{AK}$ Zulhisyam (2014).The future of the Malaysian beefindustry.J. Trop. Resour. Sustain. Sci. 2: 23-29.

Khandoker MAMY, $\mathrm{K}$ Imai, $\mathrm{T}$ Takahashi and $\mathrm{K}$ Hashizume (2001). Role ofgelatinase on follicular atresia in the bovine ovary.Biol. Reprod. 65:726-732.

Khandoker MAMY, N Jahan, LY Asad, SAM Hoque and $S$ Ahmed (2011).Qualitative and quantitative analysis of buffalo ovaries, follicles and oocytes in viewof in vitro production of embryos.Bang. J. Anim. Sci.409:23-27.

Kumar N, S Paramasivan, P Sood and M Singh (2004). Micrometry of different category oocytes recovered from goat ovaries. Ind. J. Anim. Sci. 74: 259-260.

Microsoft (2007). Microsoft Excel (computer software). Redmond, Washington: Microsoft.

Mondal MA, MAMY Khandoker, MA Mondal, AHMS Rahman, AS Apu and S Pervage (2008). In vitro production of goat embryos in Bangladesh. Bang. J. Anim. Sci. 37: 1-9.

Nandi S, MS Chauhan and P Palta (2000). Effect of a corpus luteum on the recovery and developmental potential of buffalo oocytes. Vet. Res.147: 580-581.

Nowshari MA (2004). The effect of harvesting technique on efficiency of oocyte collection and different maturation media on the nuclear maturation of oocytesin camels (Camelus dromedaries).Therioge-nology. 63: 2471-2481.

Saha S, MAMY Khandoker, LY Asad, , AMMT Reza and A Hoque (2014). Effect of fresh and frozen semen on in vitro fertilization and subsequent development of goat embryos. Iran. J. Appl. Anim.Sci. 4: 325-330.

Shirazi A, N Shams-Esfandabadi and SM Hossein (2005). A comparison of two recoverymethods of ovine oocytes for in vitro maturation.Small Rumin.Res. 58:283-286.

Sianturi RG (2001). In vitro production of embryos from abattoir-derived cattle oocytes.Master of Science Thesis.Universiti Putra Malaysia.

Singh LR, KS Rao and KM Mohan (2013). Oocyte retrieval methods, grade and percentage of oocytes in goats. Int. J. Mol. Vet. Res.3: 4-6.

Sirard MA and P Blondin (1996).Oocyte maturation and IVF in cattle.Anim.Reprod. Sci. 42: 417426.

Sreenivas D, DSVGK Kaladhar, NS Yarla, VM Thomas, A PalniSamy, VR Vadlapudi and R Preethi (2014).In vitro production of sheep embryos in CR1aa medium supplemented with L-Ascorbic Acid. J Tissue Sci Eng. 5: 131.

Wang ZG, ZR Xu and SD Yu (2007). Effects of oocyte collection techniques and maturation media on in vitro maturation and subsequent embryo development in Boer goat. Czech J. Anim. Sci. 52: 21-25.

Webb R, BK Campbell, HA Garveric and JG Gong (1999). Molecular mechanisms regulating follicular recruitment and selection.J. Reprod. Fert.45:123-126.

Yang X, S Jiang and RH Foote (1993). Bovine oocyte development following different oocyte maturation and sperm capacitation procedures.Mol.Reprod. Dev. 34: 94-100. 